\title{
The effect of magnetic shielding, frequency and current on the inductance of noise suppression reactor
}

\author{
Igor Kornev ${ }^{1, *}$ and Alexander Shapovallov ${ }^{2}$ \\ ${ }^{1}$ Research and production company " Etna plus», 410040, Vishnevaya str. 11, Saratov, Russia \\ ${ }^{2}$ Saratov State University, Physical Department, 4120012. Astrakhanskaya str. 83, Saratov, Russia
}

\begin{abstract}
On the basis of integral relations, including integral equations, taking into account the influence of magnetic screens of nonlinear magnetic material on the inductance of multilayer coils, the influence of magnetic screens of material with different magnetic permeability on the inductance of multilayer coils at different values of the current flowing through the coil and for different frequencies of the signal is estimated.
\end{abstract}

\section{Introduction}

Significant miniaturization of noise suppression reactors (NSR), widely used in engineering to suppress parasitic oscillations in electric vehicle circuits, the main element of which, as most commonly, are multilayer inductance coils, is possible with the use of magnetic screens in their design [1]. However, it is necessary to take into account the possibility of their saturation during the passage of large DC currents through the inductor itself, as well as the dependence of the inductance value on the frequency of the parasitic signal passing through the coil $[2,3]$. For practical purposes, it is very important to have an assessment of the decrease in inductance value of such a design in the mode of high currents, as well as with frequency increasing, which is the subject of this work.

On the basis of the previously derived by authors relations [3, 4], is carried out the analyse of the magnetic screens from a nonlinear magnet influence on the multilayer coils inductance value and is assessed the influence of the current, flowing through the coil, and the frequency of the signal, passing through the coil on the inductance value. In this paper, the previously obtained relations are used to assess the effect of the magnitude of the DC current, flowing through a multilayer coil, on the efficiency of the magnetic screens made of materials with different magnetic permeability introduction. The analysis of the dependence of the multilayer coil with magnetic screens inductance value from the frequency, passing along its signal wire, is carried out - that is needed for further defining the NSR frequency dependence and determining the possibility of compliance with regulations for electromagnetic compatibility (EMC).

\footnotetext{
*Corresponding author: iak.etna@yandex.ru
} 

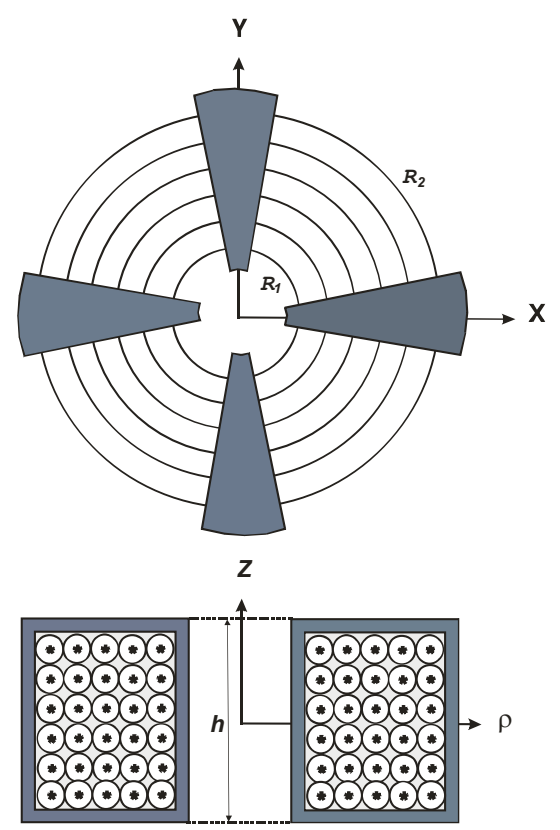

Fig. 1. The multilayer inductance coils design for which the calculations were carried out.

\section{Configuration and inductance of the noice suppression radio reactor coil}

The paper considered the coil, which design is shown in Fig.1. In her design the magnetic shields of a ferromagnetic material in the form of sectorial staples are mounted on the package of tightly compressed between each other thick copper wires in external cover. The volume of ferromagnetic material around the coil is thus determined by the thickness of the staples and the amount, they fill the angle around the circumference of the coil $(\varphi \leq 2 \pi)$. Outside the coil, the field is magnetic-dipole in nature and can be approximated by the dipole field through a given dipole magnetic moment. As a result, we calculate the energy of the magnetic field

$$
W_{H}=\mu_{0} / 2 \int_{V_{0}+V_{M}} \mu(H) H^{2} d v=L I^{2} / 2 .
$$

Here $V_{0}$ is the volume of the conductor, occupied by the current, $V_{M}$ is the volume outside the current, where the field is considered. The magnet shields is shadowing (retracting) the magnetic field, so in the first approximation, we can use as $V_{M}$ a volume containing a coil. Setting the magnetic moment $P_{M}=\mu_{0} M$, we calculate the integral (1) in the region outside this volume, since the dipole field decreases inversely to the cube of the distance, so the integral over the outer region converges and is easily calculated. The magnetic moment is determined from the condition of equality of the fields of the dipole and the field of the coil on its side surface. In a spherical coordinate system, the dipole field is defined as

$$
\begin{aligned}
& H_{r}=\frac{M}{2 \pi r^{3}} \cos (\theta), \\
& H_{\theta}=\frac{M}{2 \pi r^{3}} \sin (\theta) .
\end{aligned}
$$




\subsection{The inductance value dependence on the volume and shape of the magnetic screen}

From (1) we define the inductance value as

$$
L=\mu_{0} \int_{V_{0}+V_{M}+\Delta V} \mu(H) H^{2} d v / I^{2} .
$$

Believing that $\mu=1$ (where the magnetic substance is absent, $\mu=1$.), we obtain the inductance value of the coil without magnetic shielding. The algorithm for calculating the magnetic field is based on solving of the integro-differential equation of magnetostatics [4]. In this case, the field of the coil without a magnetic screen $\mathbf{H}_{0}$ is obtained by the BioSavard-Laplace formula by integrating (summing) the fields of each coil

$$
\mathbf{H}_{0}(\mathbf{r})=\frac{1}{4 \pi} \int_{V_{0}}\left|\mathbf{r}-\mathbf{r}^{\prime}\right|^{-1} \nabla^{\prime} \times \mathbf{J}\left(\mathbf{r}^{\prime}\right) d^{3} r^{\prime}=\frac{\nabla \times}{4 \pi} \int_{V_{0}}\left|\mathbf{r}-\mathbf{r}^{\prime}\right|^{-1} \mathbf{J}\left(\mathbf{r}^{\prime}\right) d^{3} r^{\prime}
$$

Using a cylindrical coordinate system, the integration of the field angle can be reduced in the region $(0, \pi / 2)$, and the integration in the cross-section $(\rho, z)$ is sufficient to conduct in the first quadrant. Integration on an external area is more convenient to conduct in a spherical coordinate system.

The value $\mathbf{H}$ satisfies the integro-differential equation of magnetostatics [3, 4]

$$
\mathbf{H}(\mathbf{r})=\int_{V_{0}} G\left(\mathbf{r}-\mathbf{r}^{\prime}\right) \nabla^{\prime} \times \mathbf{J}\left(\mathbf{r}^{\prime}\right) d^{3} r^{\prime}-\int_{V_{M}} G\left(\mathbf{r}-\mathbf{r}^{\prime}\right) \nabla^{\prime} F\left(\mathbf{r}^{\prime}, H\left(\mathbf{r}^{\prime}\right)\right) d^{3} r^{\prime},
$$

and the value of $\mathbf{H}_{0}$ satisfies the differential equation of magnetostatics $\nabla \times \mathbf{H}_{0}=\mathbf{J}$. Here $\mathbf{J}$ is the current density in the coil. In equation (5), the scalar value $F$ has the form

$$
F(\mathbf{r}, H(\mathbf{r}))=\nabla \cdot \mathbf{H}(\mathbf{r})=-\left\{\mu^{-1}(\mathbf{r}, H(\mathbf{r}))\left(\left[\nabla \mu(\mathbf{r}, H(\mathbf{r}))+\mu_{1}(\mathbf{r}, H(\mathbf{r})) \nabla H(\mathbf{r})\right] \cdot \mathbf{H}(\mathbf{r})\right)\right\},
$$

the gradient is taken by the first argument $\mathbf{r}$, the value $\mu_{1}(\mathbf{r}, H(\mathbf{r}))=(\partial / \partial H) \mu(\mathbf{r}, H(\mathbf{r}))$ is calculated at constant $\mathbf{r}$.

\subsection{The inductance value dependence on the coil alternative current frequency}

Ferromagnetism is a quantum effect and is determined by the orientation mechanism of magnetic polarization associated with magnetic domains. The frequency dependence of orientation polarization is determined by the Debye formula

$$
\mu(\mathbf{r}, \omega, H(\mathbf{r}, \omega))=1+\frac{\chi(\mathbf{r}, H(\mathbf{r}, 0))}{1+(\omega \tau)^{2}}-j \frac{\omega \chi(\mathbf{r}, H(\mathbf{r}, 0))}{1+(\omega \tau)^{2}} .
$$

Here we introduced a constant magnetic field $H(\mathbf{r}, 0)$ and took into account that magnetic permeability and magnetic susceptibility are nonlinear functions of the field. The specified scalar field is the RMS value of the vector field.

\subsection{The inductance value dependence on the DC current in the coil}

IDE (5) can be considered at nonlinear dependence of magnetic permeability (7) on the field. In a homogeneous sample, the magnetization curve $\mathbf{B}=\mathbf{B}(\mathbf{H})$, or for scalar quantities $B=B(H)$ is known. The value of $P_{M}=B-\mu_{0} H$ we will approximate by sigmoidal function in the form

$$
P_{M}(H)=B(H)-\mu_{0} H=2 \pi^{-1} P_{M}(0) \operatorname{arcth}(H / \bar{H}),
$$


where $\bar{H}$ is the saturation field, $P_{M}(0)$ is the magnetic moment of saturation. For magnetic permeability we have approximation

$$
\mu(H)=1+\chi(H)=1+2 \pi^{-1} P_{m}(0) /\left[\left(\mu_{0} \bar{H}\right)\left(1+(H / \bar{H})^{2}\right)\right] .
$$

Setting the current density $J$ and the current $I$ in the coil, we can determine from (5) the configuration of the magnetic field at a certain value of the magnetic permeability. This value must be specified by the formula (9) and then it must be solved again the equation (5). The process continues iteratively until the convergence. The inductance value is estimated by the formula (4).

\section{Numerical study of frequency and nonlinear properties}

In this work we had studied the magnetization curves of the most common electrical steels Э330A and $Э 41$, and cast iron, and also is carried out the numerical modelling of the characteristics of the inductors with shields made of these materials. For these inductors were formulated the dependences of inductance value changes of the value flowing through the coil, the DC bias for different materials of screen different (in relation to the maximum theoretically feasible) amounts of ferromagnetic material around the coil. Numerical evaluation of reduction of inductance value while the spurious signal frequency increasing. The dependence of the inductance value of the coil with magnetic screens in relation to the coil without screens on the DC bias current is shown in Fig.2. The calculation was carried out for a coil of 30 turns ( 5 in radius, 6 along the axis), an inner diameter of $200 \mathrm{~mm}$ and a wire with an outer diameter of $15 \mathrm{~mm}$.

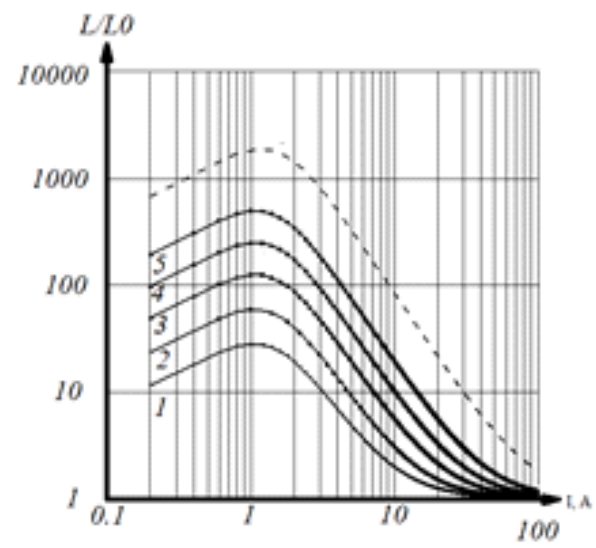

Fig. 2. The dependence of $\mathrm{L} / \mathrm{L}_{0}$ ration of the coil current $I(A)$ for different filling volume by the screen material: $\varphi=\pi / 2, \mathrm{t}=8$ (1), 16 (2), 32 (3) $\mathrm{mm} ; \mathrm{t}=32, \varphi=\pi(4) \varphi=2 \pi(5) ; \varphi=2 \pi, t=100 \mathrm{~mm}$ Curves 5 and 7 are constructed taking into account the inter-turn capacities.

To account the frequency dependence of inductance value, it is convenient to obtain the value of the effective magnetic permeability. This value can be determined on direct current as $\mu_{e f}=L / L_{0}$. At high frequencies the current does not flow through the hool cross-section of the coil wire, but only through a thin skin layer, causing the inductance value to drop. Such a drop for $L_{0}$ does not exceed $10 \%$ [5] and is small compared to the drop in inductance $L$ due to the dispersion of the magnetic permeability (7), if the fill factor of the magnet is not small. We further believe that [6]

$$
\mu_{e f}(\omega)=1+\frac{2 \pi^{-1} P_{M}(0)}{\left[1+(\omega \tau)^{2}\right]\left(\mu_{0} \bar{H}\right)\left(1+(H / \bar{H})^{2}\right)},
$$

that allows us to determine the frequency properties of the inductance (Fig. 3). 


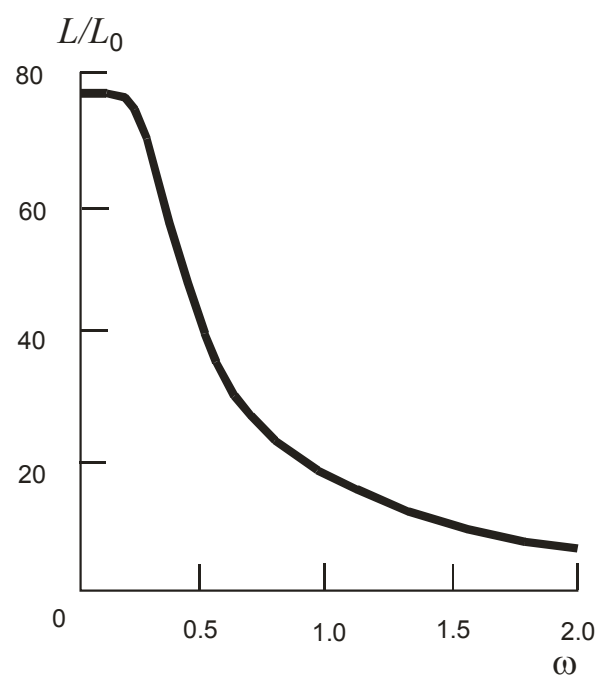

Fig. 3. The relative dependence of inductance from circular frequency $\omega(\mathrm{MHz})$ at $\mu_{e f}=77.0$.

An important parameter here is the relaxation time $\tau=1 / \omega_{c}$, which determines the quality of the material. In these calculations was taken $\omega_{c}=0.5 \mathrm{MHz}$. Ferrites have better parameters in the high-frequency region in comparison with steel, so it is advisable to use ferrite magnetic screens to improve the frequency properties.

\section{Conclusions}

In this paper, previously developed by the authors mathematical approaches are used to assess the influence of magnetic screens, made of materials with different values of magnetic permeability. The analysis of influence on multi-layered coil inductance value of flowing along it direct current value - for various values of passing it parasitic signal frequency is carried out. Here we used integro-differential equations with respect to the magnetic field. You can use similar equations with respect to the vector-potential or magnetization [6].

It is shown that with an increasing of the coil with screens current, the inductance value of the coil falls, and at a current of 100 A does not exceed the inductance of the coil without winding ferromagnetic materials more than 2 times. It is also shown that the inductance value decreases with frequency, and at a frequency of about $1 \mathrm{MHz}$ and a low current also does not exceed the inductance of the coil without screens more than several times. Much better results can be obtained by using conventional steel as a magnetic shield material, which is characterized not only by lower values of magnetic permeability in the absence of high currents, but also by a lower slope of the magnetic permeability dependence on the value of the current, flowing through the multilayer coil.

\section{References}

1. A.V. Kozlov, I.A. Kornev, S.R. Aminev, Device for suppressing interference with the operation of the electric vehicle, Patent 2013147714/07 (138345, Russian Federation, 28.10.2013) [In Russian]

2. V. M. Nosachev. I. A. Kornev, A. I. Timofeev, V. Ya. Yavchunovsky, Questions of applied physics [Voprosy pricladnoi fiziki], Saratov Univ. Publishing House, 21, 10 [In Russian] (2014) 
3. M.V. Davidovich, A.V. Kozlov, Physics of wave processes and radio engineering systems [Fizika volnovyh processov i radiotekhnicheskie sistemy], 13, 2, 46 [In Russian] (2010)

4. M. V. Davidovich, A. I.Timofeev, I. A.Kornev, V. Ya. Yavchunovsky, Izvestiya Saratov Univ. New series. Series: Physics, 16, 1, 33 [In Russian] (2016)

5. P.L. Kalantarov, L.A. Cejtlin, Calculation of inductances, Leningrad, Energoatomizdat [In Russian] (1986)

6. L. D. Gol'dshtejn, N. V. Zernov, Electromagnetic waves, Moscow, Sov. Radio [In Russian] (1971)

7. P. P. Silvester, L. R. Ferrari, Finite elements for electrical engineers. 3th ed, Cambridge: Cambridge University Press (2003) 